\title{
A visible light-curable yet visible wavelength-transparent resin for stereolithography 3D printing
}

\author{
Hong Key Park', Mikyung Shin (10', Bongkyun Kim², Jin Woo Park² and Haeshin Lee (1)
}

\begin{abstract}
Herein, a new polymeric resin for stereolithography (SLA) three-dimensional printing (SLA-3DP) is reported. An ultraviolet (UV) or visible (VIS) light source is critical for SLA printing technology. UV light can be used to manufacture 3D objects in SLA-3DP, but there are significant occupational safety and health issues (particularly for eyes). These issues prevent the widespread use of SLA-3DP at home or in the office. Through the use of VIS light, the safety and health issues can largely be solved, but only non-transparent 3D objects can be manufactured, which prevents the application of 3DP to the production of various common transparent consumer products. For these reasons, we developed a VIS light-curable yet visibly transparent resin for SLA-3DP, which also retains UV curability. The key was to identify the photoinitiator diphenyl(2,4,6-trimethylbenzoyl)phosphine oxide (DPTBP). DPTBP was originally designed as a UV photoinitiator, but we found that VIS light irradiation is sufficient to split DPTBP and generate radicals due to its slight VIS light absorption up to $420 \mathrm{~nm}$. The cured resin displays high transparency and beautiful transparent colors by incorporating various dyes; additionally, its mechanical properties are superior to those of commercial resins (Arario 410) and photoinitiators (Irgacure 2959).
\end{abstract}

\section{Introduction}

The additive manufacturing technique commonly known as three-dimensional printing (3DP) produces solid objects from reagent materials for various applications ${ }^{1}$, such as consumer products, patient-specific medical/dental devices, and microfluidic devices ${ }^{2-5}$. Among the various operating modes of $3 \mathrm{D}$ printers ${ }^{6-14}$, stereolithography (SLA) printing is a popular method that utilizes UV or VIS light sources to cure a thin layer of liquid resin via layer-by-layer photopolymerization to construct an object. SLA printing is popular because of its time efficiency, high resolution, and easy accessibility; various polymeric resins, including acrylates and urethanes, have

\footnotetext{
Correspondence: Haeshin Lee (haeshin@kaist.ac.kr)

'Department of Chemistry, Korea Advanced Institute of Science and Technology (KAIST), 291 University Rd., Daejeon 34141, South Korea

${ }^{2}$ BioActs Co. Ltd., DK Tower 10th FL., 595th Street 9, Cheongneung-daero, Namdong-gu, Incheon 21688, South Korea

These authors contributed equally: Hong Key Park, Mikyung Shin.
}

been used to make microstructures, hydrogels, biodegradable bone ingrowth scaffolds, mandibular reconstructions, etc ${ }^{15-18}$.

Objects exhibiting both optical transparency and mechanical hardness have been a challenging problem in SLA-3DP. Printed objects with mechanical properties similar to consumer products are mostly non-transparent or partially transparent (i.e., translucent). Constructs exhibiting superior optical transparency use poly(ethylene glycol) (PEG) acrylates or their related acrylate resins ${ }^{19}$. However, these have very soft mechanical properties, limiting their applications to tissue engineering, cell culture, or drug delivery. Thus, PEG-based objects are generally not suitable for consumer products, such as toys or industrial prototypes. In addition, to cure these PEGbased materials, VIS light cannot be used as a light source; instead, high-energy UV light must be used. To date, polymer resins that provide objects with both transparency and strong mechanical properties after printing with

\section{(c) The Author(s) 2018}

(c) (i) Open Access This article is licensed under a Creative Commons Attribution 4.0 International License, which permits use, sharing, adaptation, distribution and reproduction cc) in any medium or format, as long as you give appropriate credit to the original author(s) and the source, provide a link to the Creative Commons license, and indicate if changes were made. The images or other third party material in this article are included in the article's Creative Commons license, unless indicated otherwise in a credit line to the material. If material is not included in the article's Creative Commons license and your intended use is not permitted by statutory regulation or exceeds the permitted use, you will need to obtain permission directly from the copyright holder. To view a copy of this license, visit http://creativecommons.org/licenses/by/4.0/. 
VIS light have not been developed. The use of VIS light is safe and convenient and reduces the risks of eye damage due to unintentional exposure. It is also energy efficient. Thus, VIS light SLA-3DP is particularly desirable for home, office, and commercial implementation. For instance, the US Occupational Safety and Health Administration provides a guideline for workers who use UV lamps and requires protective eyeglasses and monitoring of radiation for safety ${ }^{20}$.

Herein, we developed a resin for SLA-3DP that is curable with both UV and VIS light. The key was to identify a photoinitiator, diphenyl(2,4,6-trimethylbenzoyl)phosphine oxide (DPTBP), that can be activated by a VIS light source but is nearly colorless. Although DPTBP is currently used as a photoinitiator, existing resins always require mixtures of two or three photoinitiators ${ }^{21,22}$, which results in a non-transparent deep-yellow color in the final printed objects. In this study, we propose that the use of only a single photoinitiator, i.e., DPTBP, is sufficient to generate unprecedentedly transparent objects via the SLA 3DP technique. Along with the identified photoinitiator DPTBP, the other polymerizable components are pentaerythritol tetraacrylate (PETA) and 1,6-hexanediol diacrylate (HDA). PETA is a four-arm acrylic monomer, i.e., it has four acrylic groups that can react with PETA or HDA for solidification. Depending on the ratio between PETA and HDA, the mechanical properties can be controlled ${ }^{23}$. Similar to 3D printed PEG-based hydrogels, the printed objects shown in this report are transparent. However, unlike typical 3D hydrogel objects, they are mechanically hard (with a maximum load of 330 $\mathrm{N}$ for $\left.10 \times 5 \times 1 \mathrm{~cm}^{3}\right)$ and superior to a conventional SLA resin (maximum load of $220 \mathrm{~N}$ ) made with Irgacure 2959, a well-known UV-curable photoinitiator. For simplicity, the complicated resin formulation of DPTBP/PETA/HDA is named "UVIStar" (UV-VIS-curable transparent resin). Mixing a suitable colored dye with UVIStar results in esthetically beautiful objects and may demonstrate the practical utility of SLA-3DP in many applications.

\section{Materials and methods Materials}

PETA, DPTBP $(\geq 97 \%)$, HDA $(\geq 80 \%)$, rhodamine 123 $(\geq 85 \%)$, rhodamine B ( $\geq 95 \%)$, tetrabromophenol blue (dye $85 \%$ ), ethanol (absolute analysis grade, EMD Millipore, USA), and dimethyl sulfoxide ( $>99 \%)$ were purchased from Sigma-Aldrich (USA). Irgacure 2959 (2-hydroxy-1-[4-(2hydroxyethoxy)phenyl]-2-methyl-1-propanone) was purchased from BASF Kaisten AG (Switzerland). The colored dyes TR Red 150, TR Orange, TR Yellow 191, TR Green, TR Blue 118, TR Navy 95, TR Violet 109, TR Bordeaux 148, and TR Dark Blue were supplied by BioActs (South Korea). Food dyes, such as green, pink, blue, and black, were purchased from Bowon Food (South Korea). The Arario 410 resin and SLA 3D printer were purchased from Carima, Inc. (Master Plus J model, South Korea). The Arario 410 resin contains the diglycidyl ether of bisphenol (20-40\%), acryloyl morpholine (10-20\%), tripropylene glycol diacrylate (20-40\%), 1-hydroxycyclohexyl phenyl ketone (3-7\%), diphenylphosphine oxide (1-3\%), and 2-methyl-4-2-morpholinopropiophenone (1-3\%).

\section{Preparation of UVIStar}

The UVIStar resin was prepared using the following mixture solution (300 mL basis). First, $1471 \mathrm{mg}$ of DPTBP was dissolved in $250 \mathrm{~mL}$ of PETA. Subsequently, $24.8 \mathrm{~mL}$ of HDA was added to the DPTBP solution. The reaction container was wrapped in aluminum foil and placed on a shaker (SH30, FINEPCR, South Korea) at $230 \mathrm{rpm}$ at room temperature overnight. The resin was cured utilizing a commercial compact fluorescent lamp (TEMPAT work/clamp-lamp E36 KS2042T/01, China), a UV lamp with a wavelength $(\lambda)$ range from 300 to $390 \mathrm{~nm}\left(\lambda_{\max }=\right.$ $365 \mathrm{~nm}$ ) (RX-H300C, Raynics, South Korea), or a VIS light SLA-type 3D printer (Master Plus J, Carima, Seoul, South Korea) with a printer setting of $50 \mathrm{~s}$ of light exposure per layer $(100 \mu \mathrm{m}$ of $3 \mathrm{D}$ model height $)$ of product.

\section{D object modeling and data transmission to SLA 3D printer}

The 3D printed products in this study were designed using the commercial 3D modeling program Rhino 3D (Rhinoceros version 5 SR 12, USA). However, any 3D modeling software that generates a.STL file format can be used. The .STL file was sectioned using the StickPlus Rhino 3D plugin, and the data were transmitted to the 3D printer, resulting in a solid product of the 3D model.

\section{Physical characterization of UVIStar resins}

First, the transparency of UVIStar was evaluated by an UV-Vis spectrophotometer (HP-8453, Hewlett-Packard, USA). The UV-Vis absorption spectra of the UVIStar solution (diluted two-fold with HDA) and solution of a conventional resin, Arario 410, were recorded using a nanocuvette (sample volume of approximately $5 \mu \mathrm{L}$, Nano stick-S, Scinco, South Korea). The absorbance at $600 \mathrm{~nm}$ was used to compare the transparency (i.e., turbidity) of the solutions. In addition, to confirm the visual transparency against the word "KAIST" printed on paper, cured UVIStar samples with dimensions of $100 \mathrm{~mm} \times 10$ $\mathrm{mm} \times \mathrm{T}$ (thickness $=1,1.5$, and $2 \mathrm{~mm}$ ) were produced from a VIS light SLA-type 3D printer (Master Plus J, Carima, South Korea) and printed in Master Plus J (Carima Inc., S. Korea). To characterize the photoinitiators DPTBP (0.4 wt\% in ethanol) and Irgacure 2959 (0.1 wt\% in ethanol), UV-Vis spectra were also recorded in the wavelength range from 200 to $500 \mathrm{~nm}$. 


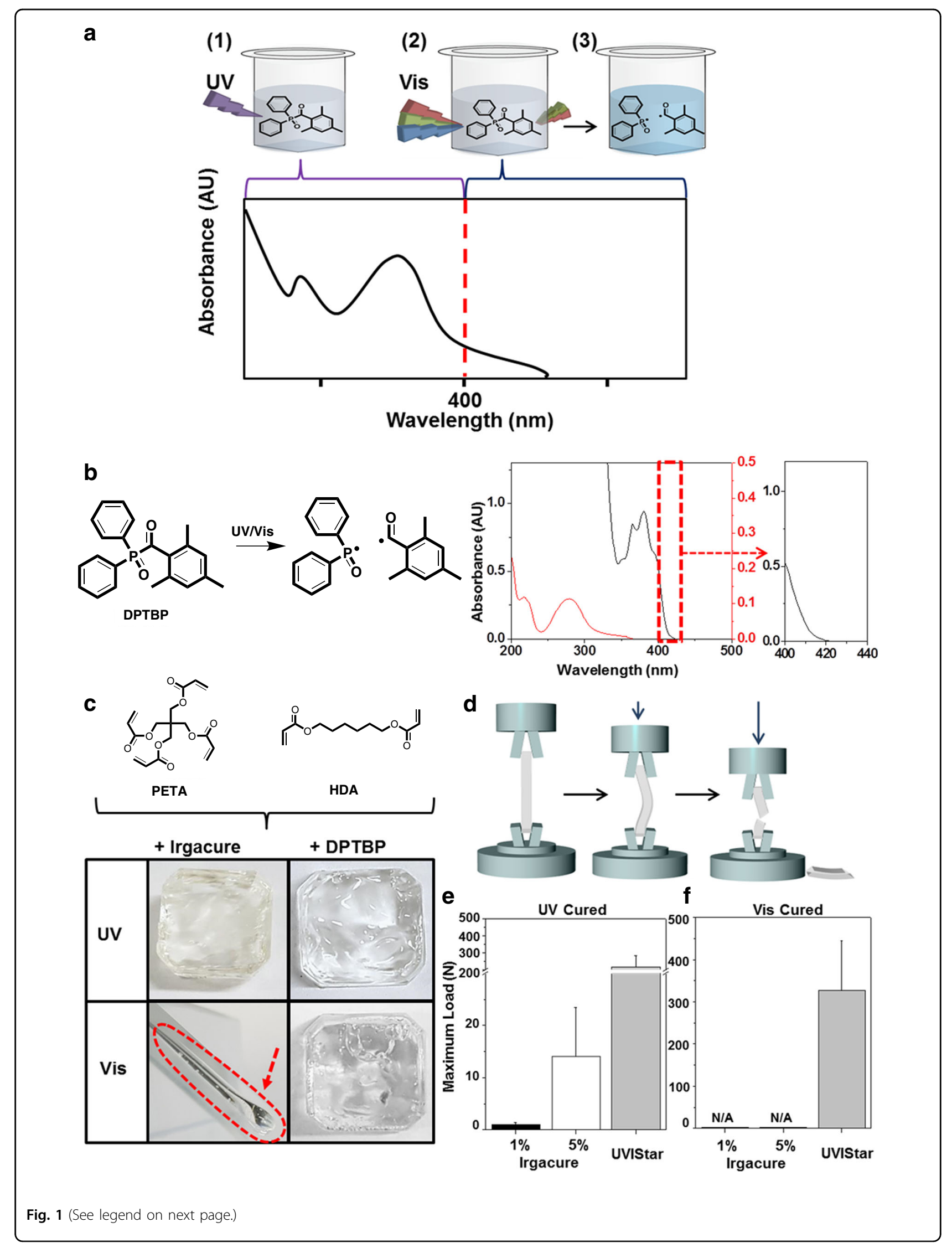


(see figure on previous page)

Fig. 1 a Schematic presentation of the requirements for a photoinitiator for a VIS light-curable yet VIS wavelength-transparent 3DP resin. b Photoinduced cleavage reaction of diphenyl(2,4,6-trimethlbenzoyl) phosphine oxide (DPTBP, left) and UVNIS absorption spectra (right panel) of DPTBP (black) and transparent UV-curable Irgacure 2959 (red). The red box represents the weak VIS wavelength absorbance of DPTBP from 400 to $420 \mathrm{~nm}$. c Chemical structures of the main resin components, namely, the polymerizable monomers PETA and HDA (top). Transparency and curability of two resins of Irgacure 2959/PETA/HDA and DPTBP/PETA/HDA (UVIStar) under a UV lamp (top row) or a VIS light lamp (bottom row). The red arrow indicates the liquid state of Irgacure 2959 resin under a VIS light source. d-f Schematic representation of the compression test for maximum load analysis (d). Comparison of the maximum load of UV (e) or VIS light (f)-cured objects for resins with 1 wt\% (black bar) and 5 wt\% (white) Irgacure 2959 and DPTBP in UVIStar (gray)

Second, compression tests were performed on cured UVIStar products with dimensions of $100 \mathrm{~mm} \times 10 \times 1$ $\mathrm{mm}$ (VIS light-curable samples were made with Master Plus J, Carima, South Korea) using a universal testing machine (UTM, Instron 5943, USA) equipped with a 500 $\mathrm{N}$ load cell, and other control UV-cured resins were prepared by UV exposure for $3 \mathrm{~min}$ followed by razor cutting to the same size. The moving speed of the UTM probe was $10 \mathrm{~mm} \mathrm{~min}^{-1}$, and the maximum compressive loading force $(\mathrm{N})$ was determined when the test samples exhibited mechanical failure. In addition, we prepared UVIStar samples to determine the resolution by creating a step thickness (height) that increased by $100 \mu \mathrm{m}$ at each step while maintaining the other dimensions $(5 \times 1.2$ $\mathrm{mm}^{2}$ ). Each step was stacked diagonally to form a stairlike shape with the $5 \mathrm{~mm}$ edges of two adjacent steps 0.3 $\mathrm{mm}$ apart and the $1.2 \mathrm{~mm}$ edges $0.15 \mathrm{~mm}$ apart. The SEM images were obtained using a Magellan 400 field-emission microscope (FEI Company, USA) at an accelerating voltage of $5 \mathrm{kV}$ for the stair-like-shaped cured UVIStar samples.

\section{Preparation of colored UVIStar resins}

Colored transparent UVIStar resins were prepared either by mixing $10 \mu \mathrm{L}$ of BioActs dye (TR Red 150, TR Orange, TR Yellow 191, TR Green, TR Blue 118, TR Navy 95, TR Violet 109, TR Bordeaux 148, and TR Dark Blue) with $5 \mathrm{~mL}$ of mixed transparent UVIStar resin solutions or by dissolving a food dye, tetrabromophenol blue, rhodamine 123 , or rhodamine $\mathrm{B}$ in DMSO $\left(0.1 \mathrm{~g} \mathrm{~mL}^{-1}\right.$ for food dye, $0.5 \mathrm{mg} \mathrm{mL}^{-1}$ for tetrabromophenol blue, 0.125 $\mathrm{mg} \mathrm{mL}^{-1}$ for rhodamine 123 , and $0.5 \mathrm{mg} \mathrm{mL}^{-1}$ for rhodamine B). The dye solutions were subsequently mixed with UVIStar resin. The UV-Vis absorption spectrum of each solution (i.e., tetrabromophenol, rhodamine 123, or rhodamine B-containing resin) was measured using an UV-Vis detector (HP-8453, Hewlett-Packard, USA). For the food dye solution, approximately $1 \mathrm{~mL}$ of dye solution was added to $100 \mathrm{~mL}$ of UVIStar resin, while the others were mixed with HDA-diluted UVIStar (two-fold) at a 1:1 volume ratio. Square-shaped cured UVIStar resin was prepared using a silicon mold with a depth of approximately $10 \mathrm{~mm}$. The BioActs dyes were used for the square-shaped cured resins and were cured with $5 \mathrm{~min}$ of UV lamp exposure. Printed chess-shaped objects with varying heights $(13 \mathrm{~mm}$ for pawns, $14 \mathrm{~mm}$ for rooks, 19 $\mathrm{mm}$ for queens and bishops, $20 \mathrm{~mm}$ for kings, and $23 \mathrm{~mm}$ for knights) were designed in the aforementioned 3D modeling program on a flat circular base with a radius of $5 \mathrm{~mm}$ and printed with a VIS light-equipped Master Plus J $3 \mathrm{D}$ printer.

\section{Results and discussion}

As mentioned in the introduction, the use of VIS light for SLA printing is advantageous over UV sources particularly due to regulations related to occupational health and safety issues. VIS light exposure does not cause-or negligibly causes-tissue damage, and SLA 3D printing machines equipped with a VIS light source are free of regulation for occupational workers and general consumers. However, photoinitiators that generate organic radicals upon exposure to VIS light exhibit strong absorption in the VIS wavelength region, resulting in the construction of translucent objects. To design a transparent resin that is effectively polymerizable using VIS light, an initiator must meet the following three criteria (Fig. 1a): (1) Most of its light absorption is located in UV regimes to minimize the intrinsic color of the photoinitiator, (2) but the right spectral edge (i.e., long wavelength region) of the initiator's absorption curve should be in the blue light region ( $>400 \mathrm{~nm}$ ) for VIS light-induced radical generation. (3) The photoinitiator should exhibit sufficient sensitivity in molecular splitting upon absorption of VIS light at the given wavelength.

After screening various photoinitiators, we identified DPTBP, the structure of which is shown in Fig. $1 \mathrm{~b}$. DPTBP satisfies the three requirements previously mentioned. The UV-Vis absorption spectrum of DPTBP shows that the majority of light absorption was detected in the UV wavelength region from 300 to $400 \mathrm{~nm}$ with a $\lambda_{\max }$ of $380 \mathrm{~nm}$. Interestingly, it also slightly absorbs VIS light from 400 to $420 \mathrm{~nm}$ (red dashed square, Fig. 1b) ${ }^{24}$. The optical properties of DPTBP are quite different from those of the well-known UV-curable photoinitiator Irgacure $2959^{25}$. The absorption spectrum for Irgacure 2959 is in the UV wavelength region (red, Fig. 1b). Typically, this 
a

[DPTBP]/[PETA]

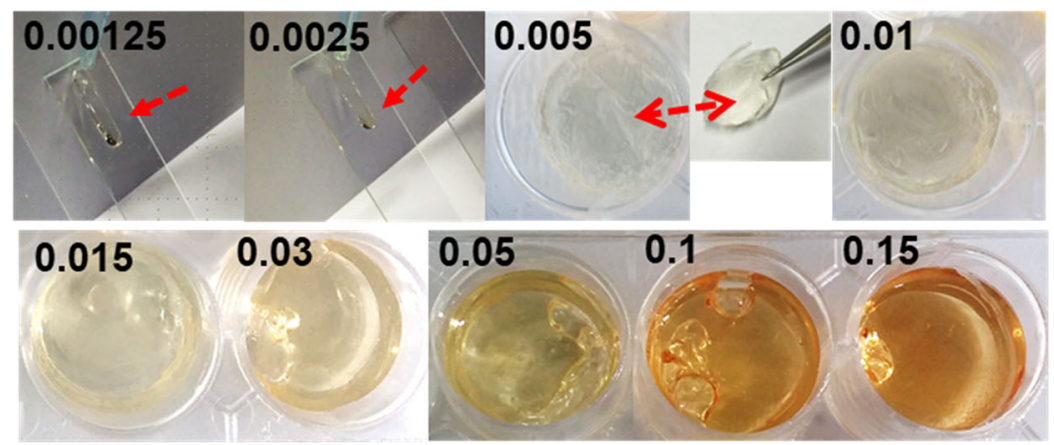

b

[HDA]/[PETA \& DPTBP]
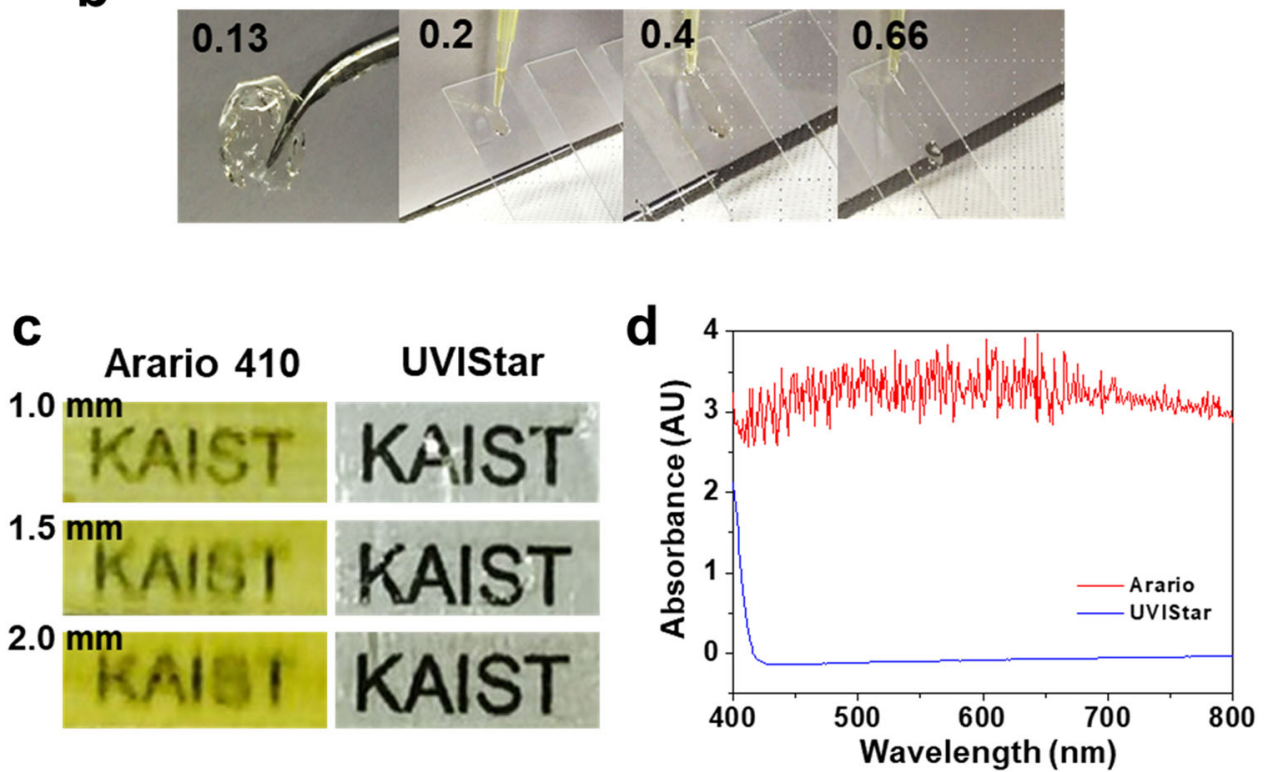

Fig. 2 a Change in transparency and color development as a function of the [DPTBP]/[PETA] molar ratios. The single-sided red arrows show noncured resin flowing down slanted glass slides, while the double-sided red arrow displays cured resin inside and outside well plate. $\mathbf{b}$ Effect of HDA addition to DPTBP/PETA formulations on curability. The distance between the VIS light lamp and resin solution was $10 \mathrm{~cm}$. The number represents the molar ratios of [HDA]/[DPTBP + PETA], and the [DPTBP]/[PETA] ratio was fixed at 0.005. c Qualitative and quantitative transparency of two VIS light-cured resins (non-transparent Arario 410 and transparent UVIStar) by overlaying on the letters "KAIST". d VIS absorption spectra of two resins: UVIStar (blue) and Arario 410 (red)

photoinitiator generates organic radicals only upon exposure to UV light, not VIS light. Thus, DPTBP satisfies the first and second requirements, which are critical for preparing a VIS light-curable resin.

Next, we focused on using the DPTBP photoinitiator to trigger radical polymerization. For this, we mixed PETA, which has four branched acrylate groups (Fig. 1c, the 1st chemical), and HDA, which has two acrylate groups (the 2nd chemical). The addition of DPTBP to PETA and HDA caused crosslinking mediated by both UV and VIS light sources (Fig. 2b, top right and bottom right panels). The cured solid objects exhibited transparency, and the DPTBP/PETA/HDA formulation is called "UVIStar", as defined in the introduction. However, under irradiation with VIS light, the Irgacure 2959/PETA/HDA formulation with $5 \mathrm{wt} \%$ Irgacure 2959 remained a transparent liquid phase that flowed down a glass slide surface tilted at an angle of $45^{\circ}$ (red arrow, Fig. 1c, left bottom panel). As expected, the Irgacure 2959/PETA/HDA solution was crosslinked under conventional UV exposure (wavelengths $(\lambda)$ from 300 to $390 \mathrm{~nm}, 5 \mathrm{~min}$ ) (Fig. 1c, left top panel).

Suitable mechanical properties of SLA 3D printed objects are essential for general consumer products. Thus, we evaluated the mechanical strength of each resin by compression of $100 \times 10 \times 1 \mathrm{~mm}^{3}$ cured objects on a 
UTM equipped with a $500 \mathrm{~N}$ load cell, as shown in Fig. 1d. The resins were clamped vertically on each side, and the upper clamp was lowered at a rate of $10 \mathrm{~mm} \mathrm{~min}^{-1}$. The maximum load $(\mathrm{N})$ that samples withstood before breaking was measured. The values for UVIStar (DPTBP/ PETA/HDA) and commercially available Irgacure 2959/ PETA/HDA cured either under UV or VIS light were compared (Fig. 1e, f). As shown in Fig. 1e, for printed UVcured objects, the maximum load was $1.1 \pm 0.4 \mathrm{~N}$ for $1 \mathrm{wt}$ $\%$ (black bar) and $14.1 \pm 9.4 \mathrm{~N}$ for $5 \mathrm{wt} \%$ (white) Irgacure 2959/PETA/HDA and $216.9 \pm 65.8 \mathrm{~N}$ for the UVIStar resin (gray bar). This compressive load is higher than that obtained using commercial UV-cured Irgacure 2959 resin. The Irgacure 2959/PETA/HDA formulation was not cured with VIS light, so a corresponding compressive force could not be determined. By contrast, the UVIStar resin was successfully cured by a VIS light source exhibiting a high compressive load $(326.3 \pm 118.0 \mathrm{~N})$ (Fig. 1f, gray bar). In addition, the curing rate of UVIStar was faster than that of the Irgacure 2959 formulation under UV radiation (data not shown). We found that $3 \mathrm{~min}$ of curing time for an object with dimensions of $100 \times 10 \times 1$ $\mathrm{mm}^{3}$ was sufficient for complete solidification when using UVIStar resin but was not enough for complete curing of the Irgacure 2959 formulation.

Due to the slight blue light absorption (i.e., yellow color) of DPTBP $(<420 \mathrm{~nm})$, concentration-dependent yellow color development was observed, as shown in Fig. 2a. The stoichiometric ratio of [DPTBP]/[PETA] was varied over nearly two orders of magnitude from 0.00125 to 0.15 , and the high photoinitiator concentration ranges, i.e., [DPTBP]/[PETA] ratios of at least 0.03 , led to a yellow color in the UVIStar resin. By contrast, [DPTBP]/[PETA] ratios lower than 0.0025 showed no VIS light curing. For VIS light curing times prolonged beyond $3 \mathrm{~h}$, the resins remained in a solution state at those stoichiometric ratios. Therefore, molar ratios of both 0.005 and 0.01 are optimal for the preparation of colorless transparent UVIStar resin. A ratio of 0.005 was used for subsequent experiments.

Printed objects made from PETA with only the DPTBP photoinitiator are very brittle due to over-crosslinking originating from the four-arm molecular structure of PETA. Thus, a molecule with a linear configuration should be added to reduce the brittleness. The aforementioned linear molecule HDA was added by adjusting the [HDA]/[DPTBP and PETA] ratios from 0.13 to 0.66 while fixing the [DPTBP]/[PETA] ratio at 0.005 (Fig. 2b). Under VIS light, the 0.13 molar ratio resin was cured, but the other resin formulations with ratios of $0.2,0.4$, and 0.66 , corresponding to higher concentrations of HDA, were not cured. Thus, the final formulation for UVIStar resin was determined as follows: $\{[\mathrm{HDA}]=0.13 /[\mathrm{PETA}=1]+[\mathrm{DPTBP}=0.005]\}$.

The optical transparency of the UVIStar resin was qualitatively demonstrated by increasing the thickness of the printed objects $(1,1.5$, and $2 \mathrm{~mm})$. The overall dimensions of the printed objects were $100 \mathrm{~mm} \times 10$ $\mathrm{mm} \times \mathrm{T} \mathrm{mm}(\mathrm{T}=1,1.5$, and $2 \mathrm{~mm})$, and the conventional VIS light-curable non-transparent resin Arario 410 and transparent UVIStar resins were used (Fig. 2c). Six printed plates were placed on top of the printed word "KAIST", and their qualitative transparency was compared. For all samples with thicknesses of 1.0, 1.5, and $2.0 \mathrm{~mm}$, the word "KAIST" was clearly VIS through the transparent printed plates (right); however, the letters were far less VIS with the conventional VIS light-curable resin (left). The nontransparent yellow color found in the VIS light-curable resin Arario 410 is primarily due to the photoinitiators diphenylphosphine oxide, 1-hydroxycyclohexyl phenyl ketone, and 2-methyl-4'-(methylthio)-2-morpholinopropiophenone $(<10 \% \mathrm{w} / \mathrm{w})^{26}$. As previously mentioned, the DPTBP photoinitiator in UVIStar exhibits minimal colorization at a [DPTBP]/[PETA] ratio of 0.005 and minimal absorbance below $420 \mathrm{~nm}$, but maintains its curability, unlike the photoinitiators in Arario 410. This crucial difference allows UVIStar resin to cure under VIS light but retain its VIS transparency. A transparency comparison of UVIStar resin and conventional Arario 410 resin was also performed via UV/Vis spectral analysis, as seen in Fig. 2d, and the UVIStar resin spectrum (blue line) showed nearly no absorbance in the VIS light range (400-700 nm).

The mechanical strength of the two cured resins was measured by compression stress tests performed by a UTM equipped with a $500 \mathrm{~N}$ load cell (Fig. 3a). The conventional Arario 410 resin showed mechanical failure at a $221.6 \pm 44 \mathrm{~N}$ compression load, but UVIStar sustained a load of $326.3 \pm 118 \mathrm{~N}$, demonstrating an approximately $50 \%$ increase. The increased resistance against compression could be attributed to the presence of the four-arm acrylic component PETA. The UVIStar resin satisfied the lowest resolution $(100 \mu \mathrm{m})$ provided by the Master Plus J model, which we used throughout this study. As shown in Fig. 3b and c, SEM images clearly showed continuous $100-\mu \mathrm{m}$ step arrays that were printed without observing any errors during machine operation. An additional advantage of UVIStar resin is the potential to create various transparent objects (similar to colored glass) simply by adding dyes. This transparent color might increase the quality of printed objects by comparing same shaped objects with non-transparent colors.

Objects with six colors, for example, red, orange, yellow, green, blue, and indigo, were produced, as shown in Fig. 3d. Dyes added to the uncured UVIStar resin showed a certain degree of diffusion, including from left to right (pink, bottom left) and from the middle to the edge (dark blue, bottom right), indicating facile control of color intensity (Fig. 3e). Finally, objects printed with colored UVIStar resins demonstrated impressive appeal due to their unique optical properties. For instance, we manufactured chess pieces 


\section{a}

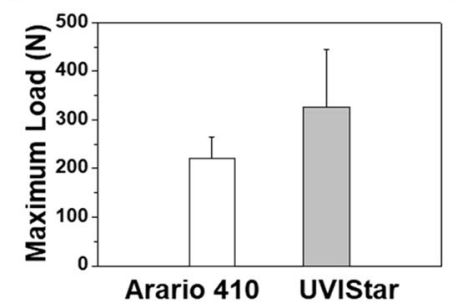

d

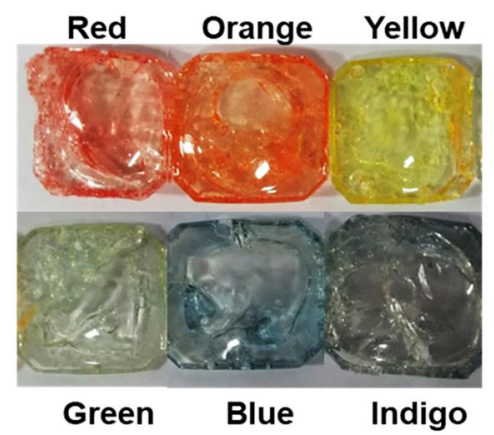

f

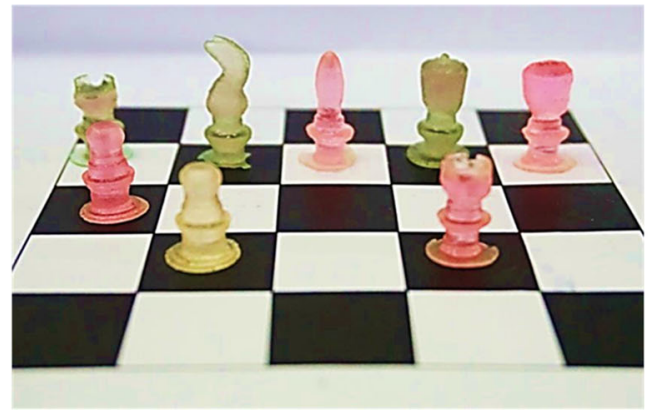

b

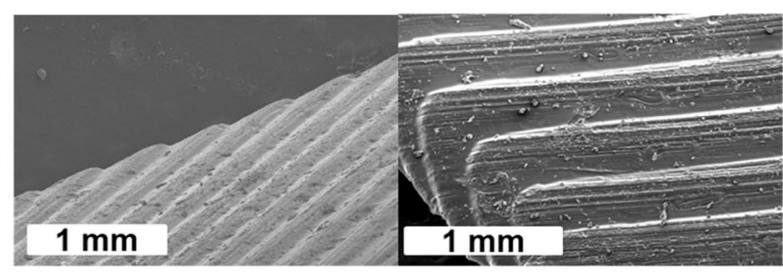

e

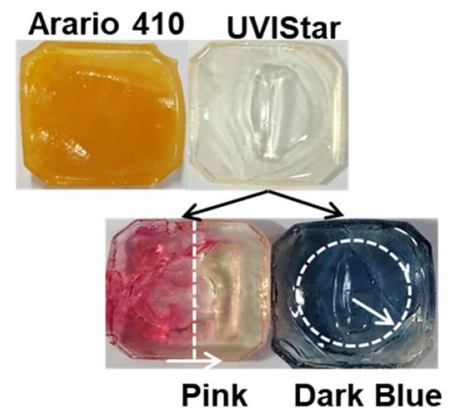

g

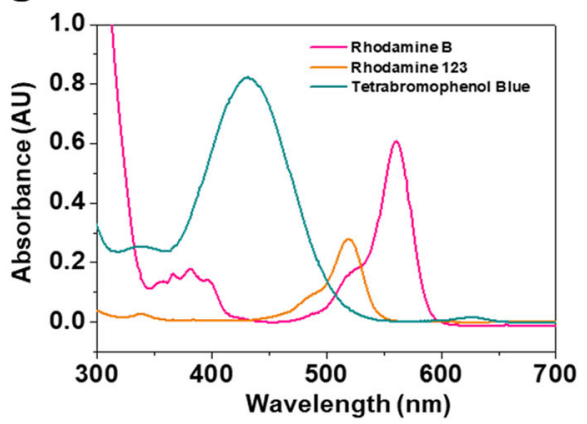

Fig. 3 a Comparative compressive force experiments for VIS light-cured conventional Arario 410 (white bar) and UVIStar resins (gray bar). Printed object dimensions are $10 \times 5 \times 1 \mathrm{~cm}^{3}$. b, c SEM images of stair-like structured cured transparent UVIStar resin from a side view (b) and a top view (c). d Photos of objects printed with UVIStar resins with various dyes included: red (top left), orange (top middle), yellow (top right), green (bottom left), blue (bottom middle), and indigo (bottom right). e Demonstration of pink dye spreading from left to right and dark blue dye spreading from the middle to the edges. $\mathbf{f}$ Various cured colored resins in chess piece shapes printed with a VIS light lamp-equipped SLA 3D printer. The chess pieces are placed on top of a mini-chessboard, demonstrating their unprecedented colorfulness. $\mathbf{g}$ Absorbance spectra of colored UVIStar resins. The turquoise line represents the spectrum of UVIStar resin with tetrabromophenol blue dye, the orange line represents the spectrum of UVIStar resin with rhodamine 123, and the pink line represents the spectrum of UVIStar with rhodamine B

with different colors (Fig. 3f). For color development, rhodamine $\mathrm{B}$, rhodamine 123 , and tetrabromophenol blue were used. The optical spectra of the three dyes are shown in Fig. $3 \mathrm{~g}: \lambda_{\max }=560 \mathrm{~nm}$ (rhodamine B), $\lambda_{\max }=$ $520 \mathrm{~nm}$ (rhodamine 123), and $\lambda_{\max }=432 \mathrm{~nm}$ (tetrabromophenol blue). The dyes were dissolved in DMSO $\left(0.1 \mathrm{mg} \mathrm{mL}^{-1}\right)$, and $1 \mu \mathrm{L}$ of each dye solution was mixed with UVIStar resin $(1 \mathrm{~mL})$. The unique transparency with or without dyes and the capability for VIS light curing demonstrated by the UVIStar resin might contribute improved practical utility in fabricating a variety of consumer products in the future. Finally, one issue remains to be resolved. There are a number of VIS light- curable resins available commercially, and component concentrations in these resins have already been optimized. However, processes for composition optimization are aimed at only their mechanical properties and curing time (i.e., printing speed), not at their optical properties, such as transparency, as demonstrated in this study. We performed additional experiments to prove that UVIStar resin is optimized regarding both mechanical and optical properties. For example, we replaced DPTBP with three other commonly used VIS light-curable photoinitiators (1-phenyl-1,2-propanedione (PPD), camphorquinone (CQ), and phenylbis (2,4,6-trimethylbenzoyl)phosphine oxide (PTBP)) at 
concentrations of $4 \mathrm{mg} \mathrm{mL}^{-1}, 2 \mathrm{mg} \mathrm{mL}^{-1}$, and $4 \mathrm{mg}$ $\mathrm{mL}^{-1}$, respectively ${ }^{27,28}$. The results showed that all the resin formulations obtained after replacing the UVIStar photoinitiator (PPD, CQ, and PTBP) exhibited a nontransparent yellow color (Supplementary Fig. S1).

\section{Conclusion}

In conclusion, we developed an SLA resin formulation that is curable under both UV and VIS light sources. The formulation consists of a DPTBP/PETA/HDA mixture at a stoichiometric ratio of 0.005:1:0.13 and is called UVIStar. UVIStar was successfully cured by UV and VIS light, showing transparency and strong mechanical strength for producing consumer objects. In particular, the photoinitiator DPTBP was crucial for the resin due to its three unique properties: (1) absorption of most UV light, (2) absorption followed by radical generation in the long wavelength region $(>400 \mathrm{~nm}$ ) for VIS curing, and (3) high sensitivity in molecular splitting. Our UVIStar products exhibited better mechanical properties and printing resolution than products made of a conventional resin (Arario 410). In addition, the cured UVIStar resin displayed beautiful translucent colors after the addition of various dyes. Our findings regarding this light-curable resin will be useful in increasing the practical utilities of SLA 3D printers in many applications.

\section{Acknowledgements}

This work was supported by the National Science Foundation of Republic of Korea: Mid-career grant (2017R1A2A1A05001047, H.L.) and the Convergence R\&D over Traditional Culture and Current Technology (2016M3C1B5906485, H.L.). The authors thank KOLON Corporation, Korea, for providing funding for this research through the KOLON-KAIST Lifestyle Innovation Center Project (LSI15-MALHS0001).

\section{Conflict of interest}

The authors declare that they have no conflict of interest.

\section{Publisher's note}

Springer Nature remains neutral with regard to jurisdictional claims in published maps and institutional affiliations.

Supplementary information is available for this paper at https://doi.org/ 10.1038/s41427-018-0021-x.

Received: 28 August 2017 Revised: 24 November 2017 Accepted: 24 December 2017.

Published online: 5 April 2018

\section{References}

1. Dimitrov, D., Schreve, K. \& de Beer, N. Advances in three dimensional printing —state of the art and future perspectives. Rapid Prototyp. J. 12, 136-147 (2006).

2. Nakayama, Y. et al. In-body tissue-engineered aortic valve (Biovalve type VII) architecture based on 3D printer molding. J. Biomed. Mater. Res. B Appl. Biomater. 103B, 1-11 (2015).

3. Kitson, P. J., Rosnes, M. H., Sans, V., Dragone, V. \& Cronin, L. Configurable 3Dprinted millifluidic and microfluidic 'lab on a chip' reactionware devices. Lab. Chip. 12, 3267-3271 (2012).

4. Comia, G., Suska, A. \& Filippini, D. PDMS lab-on-a-chip fabrication using 3D printed templates. Lab. Chip. 14, 424-430 (2014).
5. Metzger, M. C. et al. Manufacturing splints for orthognathic surgery using a three-dimensional printer. R. Oral Surg. Oral Med. Oral Pathol. Oral Radiol. Endod. 105, e1-e7 (2008).

6. Yang, S., Leong, K.F., Du, Z. \& Chua, C.-K. The design of scaffolds for use in tissue engineering. Part I. Traditional factors. Tissue Eng. 7, 679-689 (2001).

7. Dimitrov, D., van Wijck, W., Schreve, K. \& de Beer, N. Investigating the achievable accuracy of three dimensional printing. Rapid Prototyp. J. 12, 42-52 (2006).

8. Zein, I., Hutmacher, D. W., Tan, K. C. \& Teoh, S. H. Fused deposition modeling of novel scaffold architectures for tissue engineering applications. Biomaterials 23, 1169-1185 (2002).

9. Gibson, I. \& Shi, D. Material properties and fabrication parameters in selective laser sintering process. Rapid Prototyp. J. 3, 129-136 (1997).

10. Warnke, P. H. et al. Ceramic scaffolds produced by computer-assisted 3D printing and sintering: characterization and biocompatibility investigations. J. Biomed. Mater. Res. B Appl. Biomater. 93B, 212-217 (2010).

11. Kruth, J.P., Van der Schueren, B., Bonse, J. E. \& Morren, B. Basic powder metallurgical aspects in selective metal powder sintering. CIRP Ann. Manuf. Technol. 45, 183-186 (1996).

12. Li, S., Wang, C., Zhang, W. \& Li, Y. Fabrication and characterization of porous Ti6Al4V parts for biomedical applications using electron beam melting process. Mater. Lett. 63, 403-405 (2009).

13. Giordano, R. A. et al. Mechanical properties of dense polylactic acid structures fabricated by three dimensional printing. J. Biomater. Sci. Polym. Ed. 8, 63-75 (1996).

14. Park, A., Wu, B. \& Griffith, L. G. Integration of surface modification and 3D fabrication techniques to prepare patterned poly(L-lactide) substrates allowing regionally selective cell adhesion. Sci. Polym. Ed. 9, 89-110 (1998).

15. Matsuda, T., Mizutani, M. \& Arnold, S. C. Molecular design of photocurable liquid biodegradable copolymers. 1. Synth. Photo. Charact. Macromol. 33, 795-800 (2000).

16. Dhariwala, D., Hunt, E. \& Boland, T. Rapid prototyping of tissue-engineering constructs, using photopolymerizable hydrogels and stereolithography. Tissue Eng. 10, 1316-1322 (2004).

17. Cooke, M. N., Fisher, J. P., Dean, D., Rimnac, C. \& Mikos, A. G. Use of stereolithography to manufacture critical-sized 3D biodegradable scaffolds for bone ingrowth. J. Biomed. Mater. Res. B Appl. Biomater. 64B, 65-69 (2003).

18. Cohen, A., Laviv, A., Berman, P., Nashef, R. \& Abu-Tair, J. Mandibular reconstruction using stereolithographic 3-dimensional printing modeling technology. Oral Surg. Oral Med. Oral Pathol. Oral Radiol. Endod. 108, 661-666 (2009).

19. Hahn, M. S. et al. Photolithographic patterning of polyethylene glycol hydrogels. Biomaterials 27, 2519-2524 (2006).

20. Bergmanson, J. P. G. \& Söderberg, P. G. The significance of ultraviolet radiation for eye diseases. Ophthal. Physiol. Opt. 15, 83-91 (1995).

21. Manojlovic, D. et al. Effect of resin and photoinitiator on color, translucency and color stability of conventional and low-shrinkage model composites. Dent. Mater. 32, 183-191 (2016).

22. Li., J. et al. Synthesis and properties of a low-viscosity UV-curable oligomer for three-dimensional printing. Polym. Bull. 73, 571-585 (2016).

23. Roedel, M. J. The molecular structure of polyethylene. I. Chain branching in polyethylene during polymerization. J. Am. Chem. Soc. 75, 6110-6112 (1953).

24. Castelvetro, V., Molesti, M. \& Rolla, P. UV-curing of acrylic formulations by means of polymeric photoinitiators with the active 2,6-dimethylbenzoylphosphine oxide moieties pendant from a tetramethylene side chain. Chem. Phys. 203, 1486-1496 (2002).

25. Coimbra, P., Fernandes, D., Ferreira, P., Gil, M. H. \& De Sousa, H. C. Solubility of Irgacure 2959 photoinitiator in supercritical carbon dioxide: experimental determination and correlation. J. Supercrit. Fluids 45, 272-281 (2008).

26. Gan, K., Bishop, T., Lee, T.-Y., Cao, H., Diaz, M. Led curing of radiation curable floor coatings US Patent US20130224495 (2013).

27. de Oliveira, D. C. R. S. et al. Effects of different photoinitiators and reducing agents on cure efficiency and color stability of resin-based composites using different LED wavelengths. J. Dent. 43, 1565-1572 (2015).

28. Sun, G. J. \& Chae, K. H. Properties of 2,3-butanedione and 1-phenyl-1,2-propanedione as new photosensitizers for visible light cured dental resin composites. Polymer 41, 6205-6212 (2000). 